\title{
Communication
}

\section{Effect of Maxillary Implants Region and Loading Condition in the Stress Distribution of Implant-Supported Full-Arch Prosthesis: 3D-FEA}

\author{
Mateus Favero Barra Grande 1, Marcelo Lucchesi Teixeira 2, André Antônio Pelegrine ${ }^{2}$, Guilherme Da Rocha \\ Scalzer Lopes ${ }^{1}$, Julio Ferraz Campos ${ }^{1}$, and Renato Sussumu Nishioka ${ }^{2, *}$ \\ 1 Department of Dental Materials and Prosthodontics, São Paulo State University (Unesp), Institute of Science \\ and Technology, São José dos Campos - SP, Brazil , posgraduacao.ict@unesp.br \\ 2 Department of Dental Prosthodontics, São Leopoldo Mandic University, Campinas - SP, Brazil, cur- \\ so@slmandic.edu.br \\ * Correspondence: drmateusfbg@hotmail.com; Tel.:+5519982040425
}

\begin{abstract}
The effect of the different dental implants positioning region on the stress performance of the implant-supported prosthesis is not yet clear. This study evaluated the dental treatment with six dental implants in three different models and three different occlusal loading conditions, in terms of the biomechanical response of implants, prosthetic screw and maxilla, using three-dimensional finite element analysis. The finite element models were modelled containing external hexagon implants, as well as a Cobalt-Chromium superstructure. Three types of loads were applied: in the area of the central incisors, first premolar and in the second molars. For the finite element simulations, the von-Mises stress peaks in the implant and in the surrounding cortical bone were analyzed. All recorded results reported higher values for the implant-supported prosthesis in group $\mathrm{C}$ compared to the groups $\mathrm{A}$ and $\mathrm{B}$. The highest stress values, regardless the evaluated model, was in the prosthesis in group $\mathrm{C}$ and in screws, the smallest were in group $\mathrm{A}$.

In both groups, regardless of the magnitude and direction of loading, the maximum von-Mises stresses recorded for the implants and denture displacements were smaller in group A. It was concluded that the presence of more distal implants resulted in higher stress magnitude when the load was applied to the anterior region of the implant-supported prosthesis.
\end{abstract}

Keywords: Dental Implants; biomechanical phenomena; dental prosthesis; finite element analysis

\section{Introduction}

Implants with external hexagon-type connections have biomechanical characteristics with acceptable performance immediate and in the long-term follow-up. For this and other mechanical reasons, fixed prostheses on external hexagon implants are a very popular option in the treatment of edentulous patients [1].

However, for rehabilitation with an implant-supported restoration, it is necessary to have adequate bone remnant to support the masticatory loads. After tooth extraction or tooth loss, the alveolar bone undergoes a physiological remodeling process that often limits the amount of bone, especially in the anterior region of the maxilla, making rehabilitation impossible through implants in this region [2]. Thus, there are two alternatives that can provide the necessary anchorage in these areas: bone grafting or the use of long implants in the posterior region with anchorage in other portions of the bone 
tissue [3]. It is important to emphasize that the use of long implants with zygomatic anchorage, in addition to being invasive and requiring hospitalization, usually has a high cost to the patient [4].

A clinical option that overcomes these limitations would be the implant placement in the posterior region of the maxilla. However, this treatment option can present complications in the long-term due to the lack of standardization in the treatment plan [5]. The two major causes of implant failure are: peri-implantitis and occlusal overload. Both pathologies can act together or independently and cause peri-implant marginal bone loss that, in advanced cases, can lead to implant loss [6,7].

When the chewing loading mechanical stimuli are within physiological limits, they will result in the maintenance of the bone level, however, when the stimuli exceed the physiological limits, the consequence is a bone loss caused by the disorganization of the remodeling process [8]. Therefore, to avoid the marginal bone loss, it is of great importance to know how the masticatory loads can modify the biomechanical behavior of implant prosthesis [9], since the condition of the marginal bone of an implant in function is influenced by transmitted occlusal forces to him [10].

Axial loads transmit stresses along to the implant axis more homogeneously than oblique loads, being considered more friendly by the peri-implant bone tissues [11,12]. However, the positioning of implants and the infrastructure of a prosthesis on implants can influence the distribution of occlusal loads and result in a greater presence of oblique loads, which intensify the magnitude of stresses transferred to the marginal bone [13]. In order to improve the understanding of the biomechanical behavior of fixed prostheses on implants, in vitro and in silico studies have gained notoriety through bioengineering tools, such as, numerical analysis using finite element methods [14].

Numerical analysis using the finite element method allows simulating the possible stresses in the theoretical model. This methodology has the advantage of allowing simulation of various well-controlled conditions, allowing the analysis of the biomechanical behavior of implants in areas of difficult clinical access [15]. Therefore, this research aimed to evaluate the stress distribution of implant-supported prosthesis with different implants configurations and loading conditions.

\section{Materials and Methods}

To implement the methodology, a polyurethane model was made simulating an clinical condition. Then, external hexagon implants in different configurations and a metallic infrastructure was manufactured similar to the real condition when the treatment is performed in patients. After that, the computational analysis was performed simulating this reference model in three groups with different configurations of implant placement.

To perform the maxillary geometry, the polyurethane model was digitalized (Scanner trios 3, open format in STL). The 3D file was generated from the information based in the external geometry of the polyurethane model and transferred to a CAD software for the elaboration of the volumetric model. 
The STL file, despite ensuring a very precise geometric representation of bone structures, was converted using the Rhinoceros software (version 5.4.2 SR8, McNeel North America, Seattle,WA, USA), using reverse engineering tool. The fixed prosthesis on the implants were modeled with the same steps as the maxilla from the STL file generated by the CAD software. After, the 3D model was finally finished as a volumetric model (figure 1).
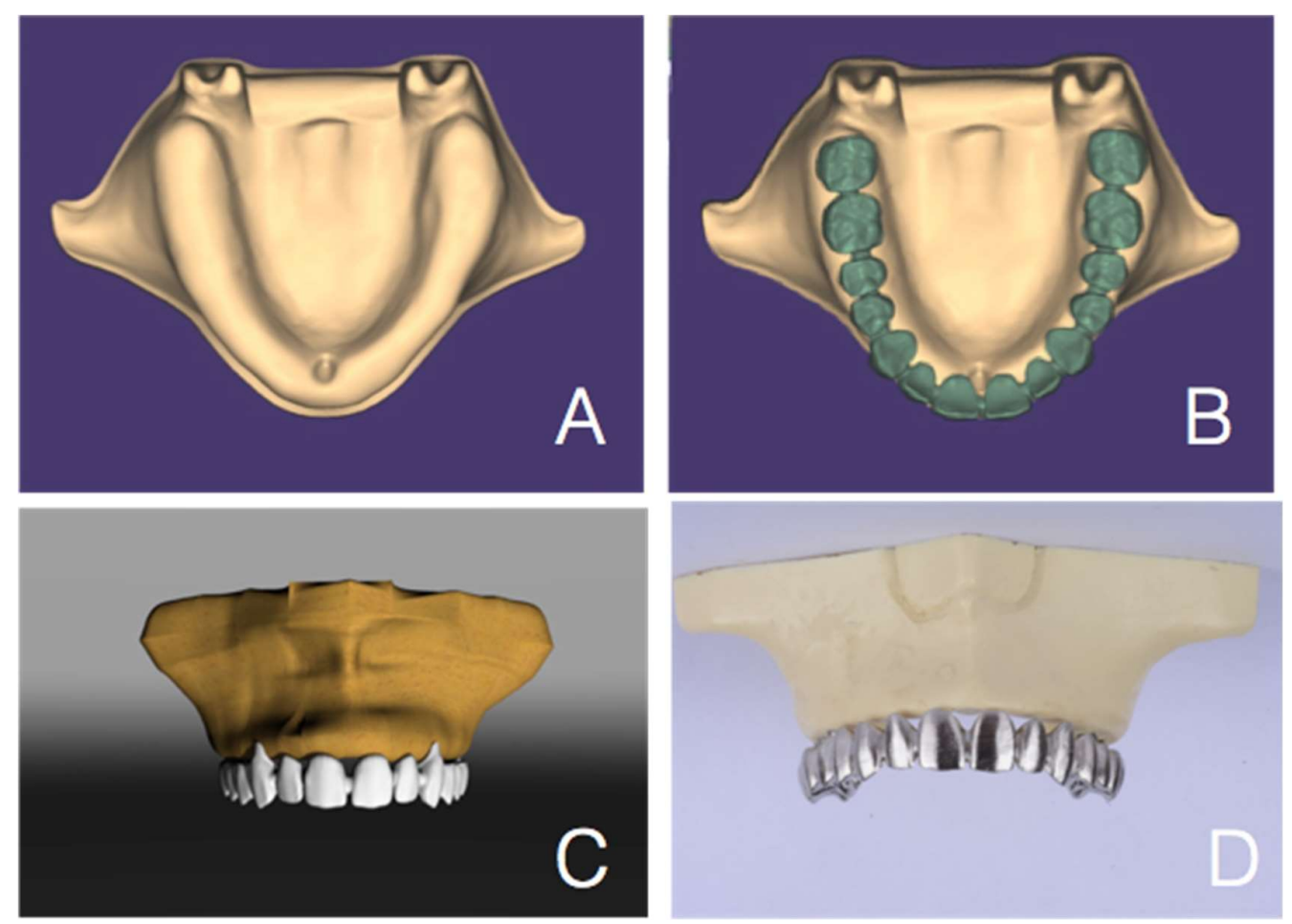

Figure 1. Figure A: shows the file generated from information from the external geometry of the pre-existing model that was transferred to a CAD software. Figure B: shows the implant-supported prosthesis modeled in CAD software. Figure C: shows the file after modeling in CAD software and the three-dimensional model was transformed into a volumetric solid and its geometry copied by the physical model. Figure D: pre-existing physical model that served as the basis for the digital archives and for the research.

After modelling, the three-dimensional model was imported into Ansys software (ANSYS 16.0, ANSYS Inc., Houston, TX, USA) in order to carry out a static structural analysis through loading and simulations. Material properties were used from software database. The geometries were renamed according to what they are representing and all structures were considered linear, homogeneous, isotropic and elastic. After checking the contact between the three-dimensional models, they are considered bonded and the number of faces tangent between two solids were adjusted with similar quantity (Figure 2). 


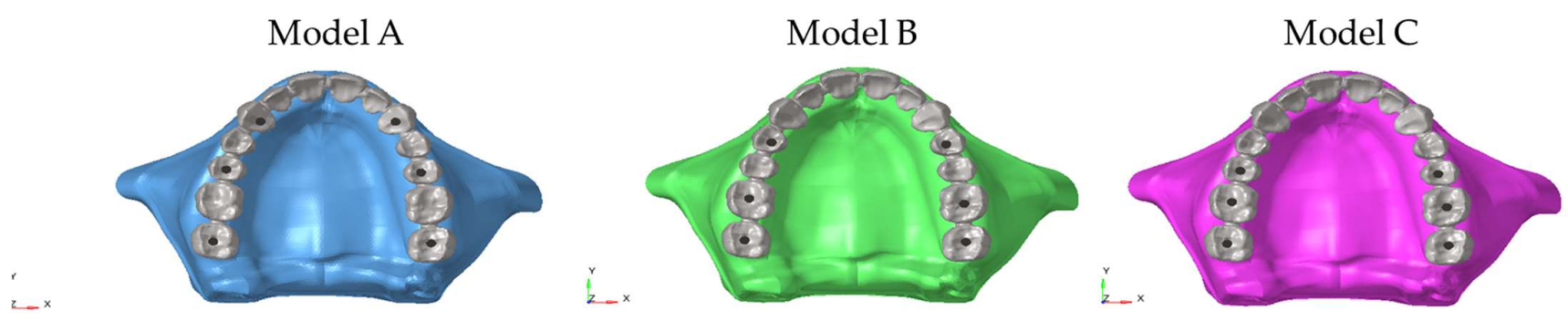

Figure 2. Group A: implants inserted in the region of canines, first premolars and second molars; Group B: implants inserted in the region of the first premolar, first molar and second molar; Group C: second premolar, first premolar and second molar.

The meshing process has been carried out automatically, in which the software allowed the refinement of the mesh created using tetrahedral elements.

For each load (axial and non-axial) an analysis configuration was used. For all configurations, the maxilla was fixed on its lower external surface simulating the support of the model in a plane. Load was defined as vector in the Z-axis direction with $300 \mathrm{~N}$ force on premolars and molars, $100 \mathrm{~N}$ on maxillary central incisors. After the simulations, solutions of von-Mises stress were obtained for the implants, screws and the maxilla model for each load (figures 3). 

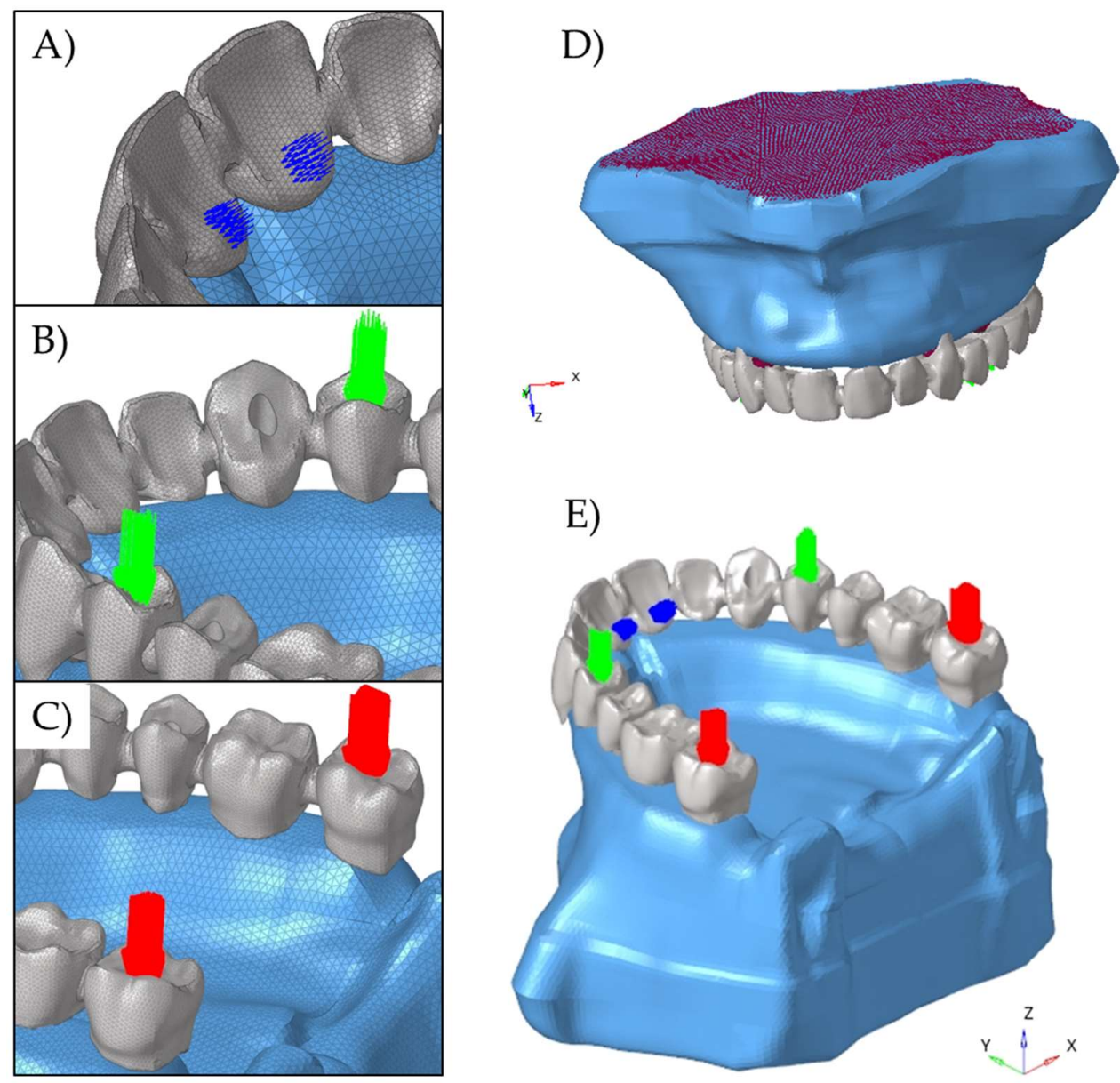

Figure 3. This figure shows the loading simulation and its regions. A) Simulation of oblique loading in central incisors, B) Simulation of axial loading in first premolars, C) Simulation of axial loading on second molars, D) Model fixation for load application with displacement 0 in $\mathrm{X}, \mathrm{Y}$ and $\mathrm{Z}$ axis. E) Perspective view of the different loading conditions.

\section{Results}

It was possible to observe for each image that the hot colors represent the zones with the highest stress concentration (von-Mises Stress), that is, regions under higher stress and for each analyzed structure.

Thus, for the oblique loads applications, it was possible to observe a lower concentration of stress and deformation in the structures furthest from the loading regions and higher stresses in the regions closer to the load application point. When the load was applied in a region of the first molar of the prosthesis, the stresses were distributed more homogeneously between the evaluated structures.

The implant-supported prosthesis was the structure with the highest stress peak for the three groups, which could be observed that in the region of the most mesial implants. However, the maxilla did not show a high magnitude of stress, in addition to the mesial region of the first implant (figures 4-6) (table 1). 
A)

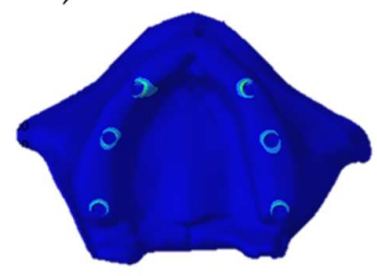

D)

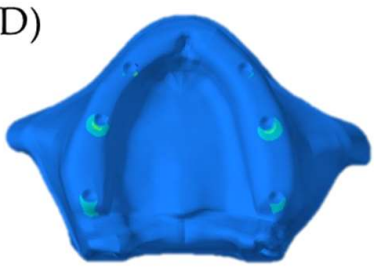

G)

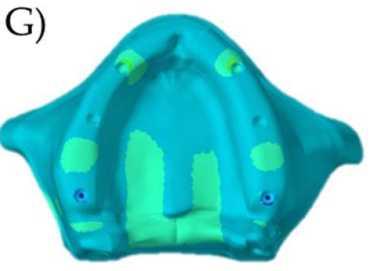

B)

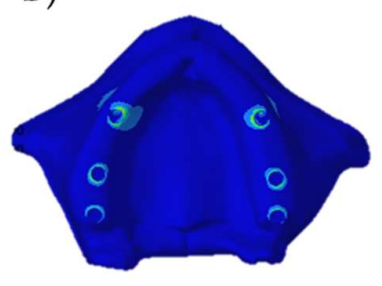

E)

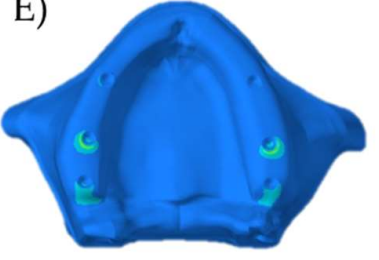

H)

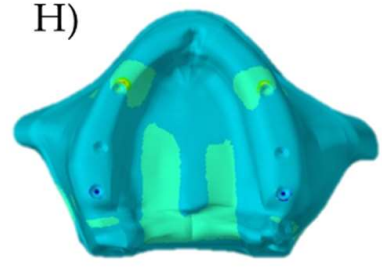

C)

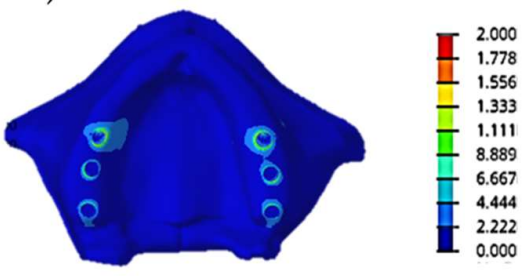

F)
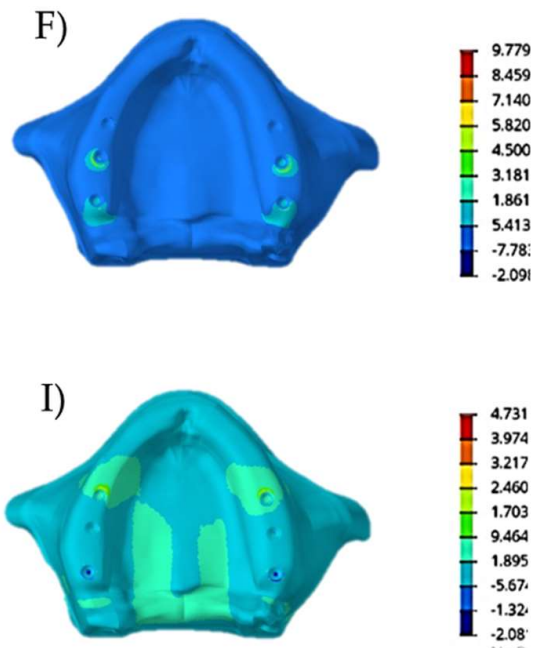

Figure 4. Von-Mises Stress (MPa) results in the bone tissue for loading in central incisors (A-C), $n$ the first premolars (D-F) and upper molars (G-I) according to the three different models.

A)

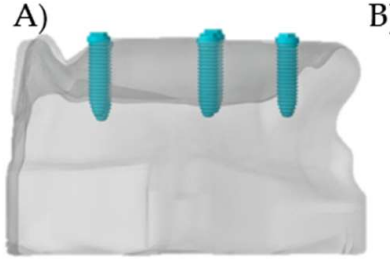

D)

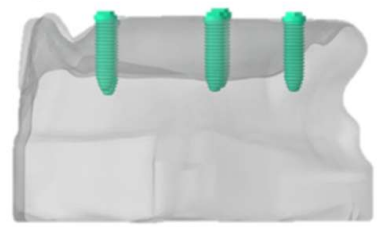

G)

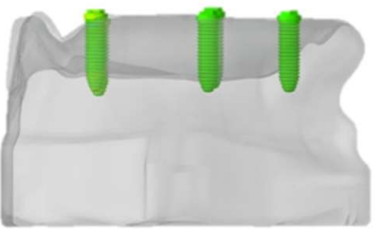

E)
B)
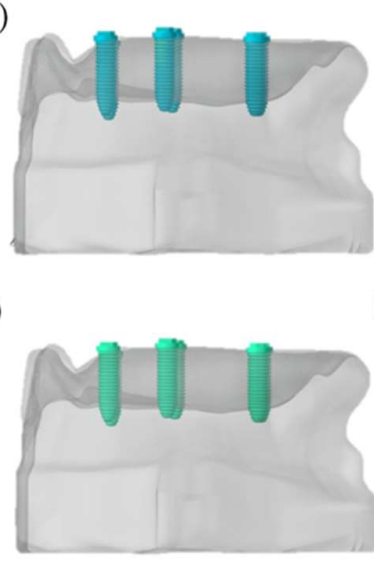

H)

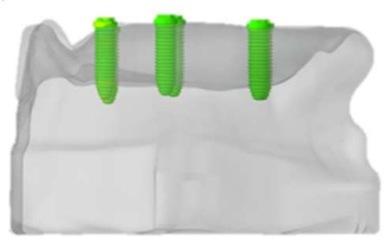

C)
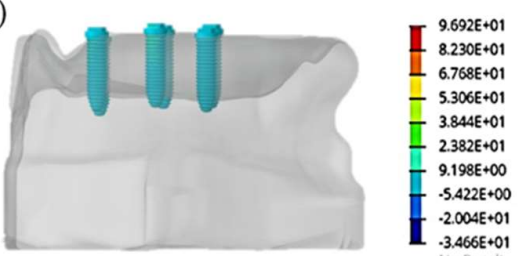

F)

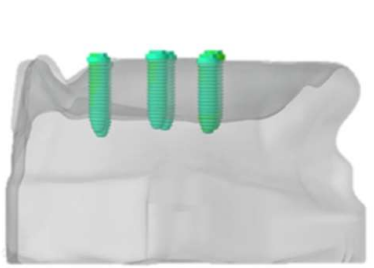

I)

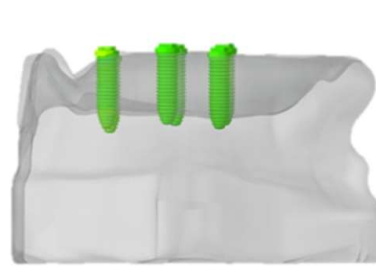

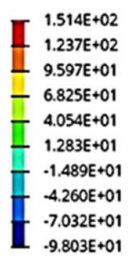

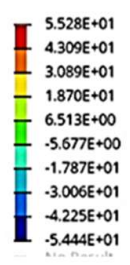

Figure 5. Von-Mises Stress (MPa) results in the dental implant for loading in central incisors (A-C), $\mathrm{n}$ the first premolars (D-F) and upper molars (G-I) according to the three different models. 
A)

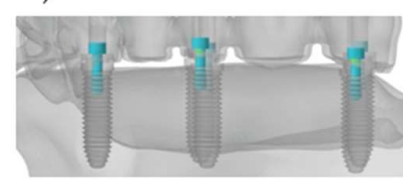

D)

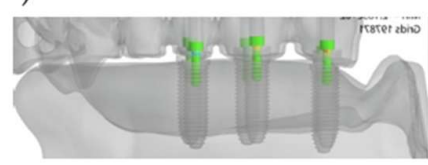

G)

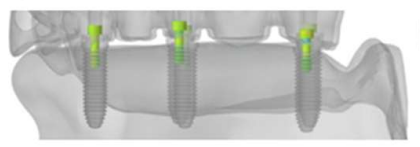

B)

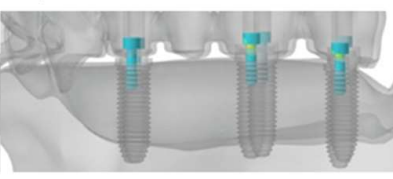

E)
C)

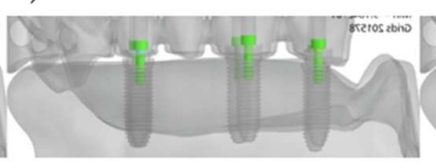

$\mathrm{H})$

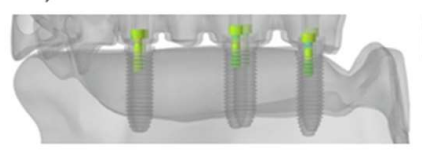

\section{C)}

F)

I)
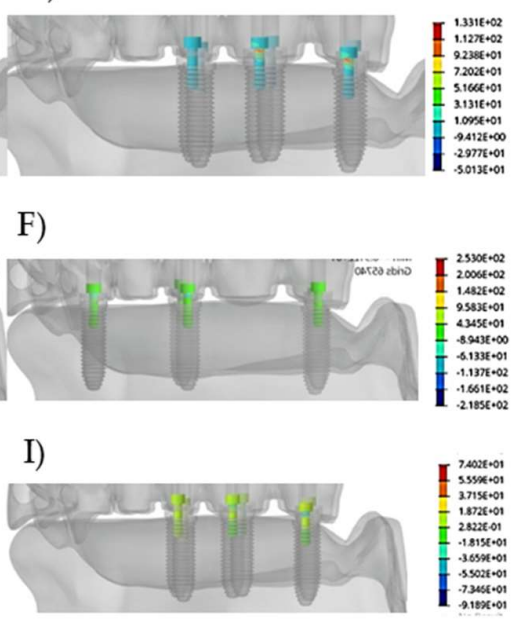

Figure 6. Von-Mises Stress (MPa) results in the prosthetic screw for loading in central incisors (A-C), the first premolars (D-F) and upper molars (G-I) according to the three different models.

Table 1. Stress peaks (MPa) calculated according the different models (A, B and C) as well as the loading region (Incisors, Premolars and Molars) for each of the evaluated region.

\begin{tabular}{ccccc}
\hline Structure & $\begin{array}{c}\text { Loading } \\
\text { condition }\end{array}$ & Model A & Model B & Model C \\
& Incisor & 65 & 13 & 44 \\
\multirow{3}{*}{ Maxilla } & Pre molars & 68 & 50 & 47 \\
& Molars & 94 & 23 & 47 \\
\hline \multirow{3}{*}{ Implant } & Incisor & 61 & 68 & 31 \\
& Pre molars & 77 & 47 & 43 \\
& Molars & 151 & 96 & 55 \\
\hline \multirow{3}{*}{ Screw } & Incisor & 68 & 96 & 57 \\
& Pre molars & 102 & 65 & 65 \\
& Molars & 253 & 133 & 74 \\
\hline \multirow{2}{*}{ Prosthesis } & Incisor & 128 & 99 & 98 \\
& Pre molars & 159 & 150 & 87 \\
\hline
\end{tabular}

Observing the deformation distribution generated in group C, it is possible to notice that the magnitude of the deformation peak is concentrated in the more mesial implants when the load applied to the centrals compared to groups A and B.

\section{Discussion}

This study evaluated the influence on external hexagon implants in three different designs of multiple implant-supported prostheses in edentulous maxillae. The results 
showed that there was a difference between groups with the calculated stress. It was also possible to observe that the greater the distance between the pontics, the greater the magnitude calculated around the implants.

Biomechanical analyzes were developed for the study of prostheses on implants. According to the literature [16] the in silico techniques, such as finite element analysis, can be used to measure bone behavior. The use of such 3D method requires prior knowledge of bone volume and mechanical properties. However, the bone tissue is not homogeneous and its physical properties vary greatly according to species, age, sex, type of bone (eg, femoral, mandibular, cortical, cancellous) and even according to location of the bone from which the sample was taken $[17,18]$.

Therefore, studies with human bone have a complexity and heterogeneity and, for ethical reasons, often hinder the development of clinical trials. Thus, through an in vitro study, a previous study evaluated the elastic modulus of an experimental polyurethane isotropic model, by means of stress tests and compared the results with those reported in the literature with bone [19]. Thus, the use of the polyurethane model in place of bone in in vitro biomechanical studies was validated. Thus, the present study used these data as a basis to manufacture the in vitro polyurethane and digital models with the properties of the physical isotropic model.

In the present results, the disposition of the implants to distal in the edentulous maxilla caused an unfavorable biomechanical behavior for the analyzed structures, in accordance with previous articles that reported a similar behavior, when the distance between the implants increased [20-22]. A previous study [23] reported that implants installed respecting the minimum space between each implant, demonstrated a better mechanical response of bone tissue to masticatory loads. Likewise, the use of six implants in group A represented an ideal distribution, which reduced the bone stress generated when applying the chewing loads.

The results obtained for the different implant positions clearly indicated that the stress and bone deformation were lower in group A compared to other groups B and C.

The stress in the bone significantly increased as the implants were installed distally to the molar region. Therefore, the more distal the configuration of the implants, the greater the distance between the anterior abutments of the implant. When the load was on the central incisors, the furthest from the first abutment of the implant in Group C, bone stress were visibly higher compared to the similar loading condition in Group A. This finding is consistent with the study of previous authors [24], who found that cantilever loading had a large effect on bone stress. Increased bone stress can increase the risk of implant loss due to bone overload [12]. The results obtained in the present study also indicated that anterior cantilever loading can negatively affect the implant stress magnitude. Although the positions of implants B and C can be considered almost similar, the loading on the central incisors increased the stress on the mesial implant in group $\mathrm{C}$ compared to group B, showing that these designs present different mechanical response.

Another novelty of the present study in relation to other reports in the literature was the verification of stress, considering a cantilever prosthesis in the anterior maxillary 
implants. Although this stress check exists in several previous studies [12, 20-26] they did not considered different implant positions as the present models. The literature [20-27] usually reports the maximum forces capable of generating mechanical problems in the bone-implant interface, however, there are no reports showing the cantilever in anterior region similar to this study.

It is noteworthy that this study was subject to some limitations, as the loading condition was simplified [27-29]. Future studies should apply different bone densities, anatomical structures such as the maxillary sinus and diversify the size and angulations of implants for this situation. Furthermore, although the experimental models were rigorously prepared and experienced dentists were involved in all procedures, the bone model is still an isotropic structure that is limited in terms of mechanical response. Therefore, it is not recommended to extrapolate these results to implant-supported prostheses in clinical situations, and further studies should be carried out to assess the effect of traction forces on the biomechanics of these prostheses. However, despite all the limitations, the present results can be evaluated to guide further studies and to elucidate how the implants region and loading condition can modify the stress magnitude.

\section{Conclusions}

Despite the study's limitation and according with the obtained results it is possible to conclude that the Model A showed the most promising mechanical behavior, while Model C and B showed the highest stress magnitude around the implants respectively. Thus, the distalized implants placement increases the stress magnitude and should not be considered as the first option during the treatment plan.

Author Contributions: Conceptualization, Mateus Barra Grande.; methodology, Renato Nishioka and Guilher Lopes.; software, Julio Campos; validation, Marcelo Teixeira. and André Pelegrine.; formal analysis, Guilherme Lopes.; investigation, Renato Nishioka; resources, Mateus Barra Grande; data curation, Renato Nishioka; writing - original draft preparation, Mateus Barra Grande ; writing-review and editing, Guilherme Lopes; project administration, Marcelo Teixeira; funding acquisition, André Pelegrine. All authors have read and agreed to the published version of the manuscript.

Funding: This research received no external funding.

Institutional Review Board Statement: Not applicable.

Informed Consent Statement: Not applicable.

Acknowledgments: The authors appreciate the support of Intraoss(R) (Warie Industrial LTDA. CNPJ: 10.615.047/0001-13. Brazil, Itaquaquecetuba - SP) by donating the implants and STL files used in this research.

Data Availability Statement: The data presented in this study are available on request from the corresponding author.

Conflicts of Interest: The authors declare no conflict of interest.

\section{References}

1. Misch, C.E.; Suzuki, J.B.; Misch-Dietsh, F.M.; Bidez, M.W. A positive correlation be- tween occlusal trauma and peri-implant bone loss: literature support.

2. Brunski, J.B.; Puleo, D.A.; Nanci, A. Biomaterials and biomechanics of oral and maxillofacial implants: current status and 
future developments. Int. J. Oral Maxillofac. Implants 2000, 15, 15-46.

3. Kizhakedathil, M.P.J.; C, S.D. Acid stable $\alpha$-amylase from Pseudomonas balearica VITPS19-Production, purification and characterization. Biotechnol. Rep. (Amst.) 2021, 30, e00603.

4. Davó, R.; Bankauskas, S.; Laurincikas, R.; Koçyigit, I.D.; Mate Sanchez de Val, J.E. Clinical performance of zygomatic implants-retrospective multicenter study. J. Clin. Med. 2020, 9, 480.

5. Kreissl, M.E.; Gerds, T.; Muche, R.; Heydecke, G.; Strub, J.R. Technical complications of implant-supported fixed partial dentures in partially edentulous cases after an average observation period of 5 years. Clin. Oral Implants Res. 2007, 18, 720726.

6. Lindhe, J.; Meyle, J.; Group D of European Workshop on Periodontology Peri-implant diseases: Consensus Report of the Sixth European Workshop on Periodontology. J. Clin. Periodontol. 2008, 35, 282-285.

7. Malchiodi, L.; Cucchi, A.; Ghensi, P.; Consonni, D.; Nocini, P.F. Influence of crown-implant ratio on implant success rates and crestal bone levels: a 36-month follow-up prospective study. Clin. Oral Implants Res. 2014, 25, 240-251.

8. Frost, H.M. Wolff's Law and bone's structural adaptations to mechanical usage: an overview for clinicians. Angle Orthod. 1994, 64, 175-188.

9. Kayumi, S.; Takayama, Y.; Yokoyama, A.; Ueda, N. Effect of bite force in occlusal adjustment of dental implants on the distribution of occlusal pressure: comparison among three bite forces in occlusal adjustment. Int. J. Implant Dent. $2015,1,14$.

11. Tribst, J.P.M.; Dal Piva, A.M. de O.; Borges, A.L.S.; Bottino, M.A. Effect of implant number and height on the biomechanics of full arch prosthesis. Braz. J. Oral Sci. 2018, 17, e18222.

12. Sahin, S.; Cehreli, M.C.; Yalçin, E. The influence of functional forces on the biomechanics of implant-supported prostheses--a review. J. Dent. 2002, 30, 271-282.

13. Isidor, F. Influence of forces on peri-implant bone. Clin. Oral Implants Res. 2006, 17 Suppl 2, 8-18.

14. Datte, C.E.; Tribst, J.P.M.; Dal Piva, A.M.O.; Nishioka, R.S.; Bottino, M.A.; Evangelhista, A.D.M.; Monteiro, F.M.M.; Borges, A.L.S. Influence of different restorative materials on the stress distribution in dental implants. J. Clin. Exp. Dent. 2018, 0-0.

15. Tribst, J.P.M.; Dal Piva, A.M. de O.; Shibli, J.A.; Borges, A.L.S.; Tango, R.N. Influence of implantoplasty on stress distribution of exposed implants at different bone insertion levels. Braz. Oral Res. 2017, 31, doi:10.1590/1807-3107bor-2017.vol31.0096.

16. Farah, J.W.; Craig, R.G.; Sikarskie, D.L. Photoelastic and finite element stress analysis of a restored axisymmetric first molar. J. Biomech. 1973, 6, 511-520.

17. Tribst, J.P.M.; Dal Piva, A.M. de O.; Lo Giudice, R.; Borges, A.L.S.; Bottino, M.A.; Epifania, E.; Ausiello, P. The influence of custom-milled framework design for an implant-supported full-arch fixed dental prosthesis: 3D-FEA sudy. Int. J. Environ. Res. Public Health 2020, 17, 4040.

18. Silveira, M.; Campaner, L.; Bottino, M.; Nishioka, R.; Borges, A.; Tribst, J.P. Influence of the dental implant number and load direction on stress distribution in a 3-unit implant-supported fixed dental prosthesis. Dent. Med. Probl. 2021, 58, 69-74.

19. O’Mahony, A.M.; Williams, J.L.; Katz, J.O.; Spencer, P. Anisotropic elastic properties of cancellous bone from a human edentulous mandible: Elastic properties of cancellous bone from an edentulous mandible. Clin. Oral Implants Res. 2000, 11, 415-421.

20. Miyashiro, M.; Suedam, V.; Moretti Neto, R.T.; Ferreira, P.M.; Rubo, J.H. Validation of an experimental polyurethane model for biomechanical studies on implant supported prosthesis--tension tests. J. Appl. Oral Sci. 2011, 19, $244-248$.

21. Bölükbaşı, N.; Yeniyol, S. Number and localization of the implants for the fixed prosthetic reconstructions: on the strain in the anterior maxillary region. Med. Eng. Phys. 2015, 37, 431-445.

22. Hekimoglu, C.; Anil, N.; Cehreli, M.C. Analysis of strain around endosseous dental implants opposing natural teeth or implants. J. Prosthet. Dent. 2004, 92, 441-446.

23. Almeida, E.O.; Rocha, E.P.; Freitas Júnior, A.C.; Anchieta, R.B.; Poveda, R.; Gupta, N.; Coelho, P.G. Tilted and short implants supporting fixed prosthesis in an atrophic maxilla: a 3D-FEA biomechanical evaluation: FEA using implant and atrophic maxilla. Clin. Implant Dent. Relat. Res. 2015, 17 Suppl 1, e332-42.

24. Liu, X.; Pang, F.; Li, Y.; Jia, H.; Cui, X.; Yue, Y.; Yang, X.; Yang, Q. Effects of different positions and angles of implants in maxillary edentulous jaw on surrounding bone stress under dynamic loading: A three-dimensional finite element analysis. Comput. Math. Methods Med. 2019, 2019, 8074096.

25. Tribst, J.P.M.; Dal Piva, A.M.O.; Bottino, M.A.; Nishioka, R.S.; Borges, A.L.S.; Özcan, M. Digital Image Correlation and finite element analysis of bone strain generated by implant-retained cantilever fixed prosthesis. Eur. J. Prosthodont. Restor. Dent. 2020, 28, 10-17.

26. Wu, A.Y.-J.; Hsu, J.-T.; Fuh, L.-J.; Huang, H.-L. Biomechanical effect of implant design on four implants supporting mandibular full-arch fixed dentures: In vitro test and finite element analysis. J. Formos. Med. Assoc. 2020, 119, 1514-1523.

27. Tribst, J.P.M.; Rodrigues, V.; Dal Piva, A.M.O.; Borges, A.L.S.; Nishioka, R.S. The importance of correct implants positioning and masticatory load direction on a fixed prosthesis. J. Clin. Exp. Dent. 2017, 0-0.

28. Tribst, J.P.M.; Rodrigues, V.A.; Borges, A.L.S.; Lima, D.R. de; Nishioka, R.S. Validation of a simplified implant-retained cantilever fixed prosthesis. Implant Dent. 2018, 27, 49-55. 
29. Datte, C.E.; Datte, F.B.; Borges, A.L.S.; Campos, J.F.; Lopes, G.D.R.S.; Tribst, J.P.M.; Nishioka, R.S. The influence of restorative material, bone height and implant system on the stress distribution of implant-supported posterior crowns. International Journal of Development Research 2021, 11, 44925-44931. 